\title{
Prevención de la depresión unipolar en España: realidad y futuro
}

\section{Prevention of unipolar depression in Spain: Current and future situation}

\begin{abstract}
Alberte Arauxo
José M. Cornes*

Hospital Universitario Gil Casares

Luis Fernández-Ríos

Universidad de Santiago

de Compostela, España
\end{abstract}

Recibido: 23 de septiembre de 2007

Revisado: 23 de octubre de 2007

Aceptado: 18 de enero de 2008

\section{Resumen}

La depresión unipolar es una de las patologías con mayor prevalencia en las sociedades occidentales. Los factores de riesgo son múltiples y muchos de ellos prevenibles. Sin embargo, en España no se toma demasiado en serio la planificación de la prevención primaria de la depresión. Concluimos que dicha situación tiene diversas motivaciones políticas y académicas. Las dos son superables, si se toma en serio la prevención de la depresión.

Palabras clave: depresión unipolar, prevención primaria, prevención universal, programas de intervención preventiva.

\section{Abstract}

Unipolar depression is one of the most common illnesses in western society. There are multiple risk factors and many of them are preventable. However, planning of primary prevention of depression is not taken very seriously in Spain. We conclude that there are various political and academic reasons for this situation. They both can be overcome if we take prevention of depression more seriously.

Key words: unipolar depression, primary prevention, universal prevention, preventive intervention programs.

* Correspondencia: José M. Cornes, Servicio de Psiquiatría. Hospital Universitario Gil Casares. C/ Choupana 15706 Santiago de Compostela. La Coruña (España). Luis Fernández-Ríos, profesor titular Universidad de Santiago de Compostela Facultad de Psicología, Departamento de Psicología Clínica y Psicobiología, Santiago de Compostela (La Coruña, España), profesor tutor de la Facultad de Psicología de la Universidad Nacional a Distancia (UNED) del Centro Asociado de La Coruña (España) y del de Pontevedra (España). Coordinador del Aula de la UNED de Lalín (Pontevedra, España). Correo electrónico: pclfr027@usc.es. 


\section{Introducción}

Parece que vivimos en la edad de la angustia, de la ansiedad o de la depresión. Sin embargo, existe una estabilidad temporal en la prevalencia de la depresión (Murphy, Sobol, Neff, Oliver y Leighton, 1984; World Health Organization, 2001, 2004). Consideramos necesario someter a una crítica constructiva lo que la psiquiatría y psicología clínica españolas hacen sobre de la prevención primaria de la depresión.

Hoy en día se está reintroduciendo la fenomenología existencial y realista en la psicología clínica y en la psiquiatría (Owen y Harland, 2007); sin embargo, si se hace con sentido común puede ser muy positivo. En la psiquiatría y en la psicología españolas se habla de hechos y valores (Baca y Lázaro, 2003). Además, se reconoce la relevancia del valor de la historia (Berrios y Olivares, 1998). Los proyectos personales requieren una axiología (Ey, 1967), así como la teoría y la práctica de la prevención. En el fondo siempre está la cuestión de comprender el encuentro cliente-técnico de salud (Barcia-Salorio, 1987), para llevar a cabo procesos de tratamiento o de prevención primaria.

Múltiples contenidos de fenomenología existencial pueden ser útiles para ayudar a la prevención de la depresión. Así mismo, se cuenta con elementos de la cultura y de la antropología cognitivo-conductual. Primero, la cultura es, al margen de lo que cada individuo piense, un sistema de significados. Segundo, la antropología cognitivoconductual aporta conocimientos relevantes para la ciencia de la prevención; la psiquiatría y la psicología cultural también. Las personas necesitan explicar la conducta; éste es el aporte más relevante de la teoría de la atribución. Los factores de riesgo de depresión los sufre la persona en un contexto sociomaterial concreto (Castilla del Pino, 1966), ya que, es en él donde se produce el desarrollo social de la personalidad (Shoda, Cervone y Downey, 2007).

La fenomenología busca responder la cuestión: ¿para qué continuar viviendo y luchando por un proyecto vital personal? Para ello, tal lugar lo mejor sea comprender, en lugar de clasificar, pues lo que el hombre hace, espera, sabe, quiere, produce e imagina constituye su modo de interpretar el mundo (Jaspers, 1977, p. 405). El sistema cultural en el que viven los individuos debe y tiene que satisfacer sus necesidades de pertenencia y de identidad. El grupo no sólo lo comprende, sino que también predice los significados de su existencia. Todo ello contribuye a controlar el proceso de existir; realzar la dignidad personal y, por último, a generar confianza en los otros. Ey (1967, pp. 36-37) hace referencia en forma muy adecuada al modelo personal del mundo que posee el ser humano. Este modo-de-estar-en-el-mundo debe poseer una estructura de valores. La psicología cognitiva actual habla de modelos mentales para referirse al resultado final de la percepción y de la comprensión del proceso de existir.

El objetivo del presente trabajo es buscar algunas descripciones y posibles explicaciones de esta decepcionante situación de la teoría y práctica de la prevención primaria de la depresión unipolar en España. Tanto la psiquiatría como la psicología se han olvidado de la prevención; no obstante, en España todavía existe un insuficiente desarrollo de los aspectos de prevención y de promoción de la salud mental (Ministerio de Sanidad y Consumo, 2007, p. 39). A pesar de la desilusionante situación, hay lugar para la esperanza; los trabajos auspiciados por la Comisión Europea acerca de Mental health promotion and mental disorder prevention (Jané-Llopis, 2006; Jané-Llopis y Anderson, 2005, 2006) son un buen ejemplo de lo que se puede hacer.

\section{Epidemiología}

La depresión es uno de los trastornos de salud más común y limitante en la Unión Europea (AyusoMateos, et al., 2001; Kessler, 2007; McCracken, et al., 2006; Tylee, 2000; Wittchen y Jacobi, 2005) y en el resto del mundo (Murray y López, 1997). La prevalencia de la depresión se estima alrededor del 17\%. En el trabajo Predict (King et al., 2006) se establece que alrededor de un quinto de las consultas de atención primaria tienen relación con sintomatología depresiva. Paykel, Bruglea y Fryers (2005) consideran que uno de 40 adultos 
de la Unión Europea presenta sintomatología depresiva en algún momento de su vida, y uno de cada 20 durante unas pocas semanas al año.

Por otra parte, la prevalencia de la ideación suicida en el European Study of the Epidemiology of Mental Disorders (ESEMED) (Bernal et al, 2007; véase también, el Journal of Clinical Psychiatry, 2007, 68 -Supplement 2- dedicado al ESEMED), es de un $7,8 \%$ en personas mayores de dieciocho años. El Longitudinal Investigation of Depression Outcomes (LIDO) es un buen ejemplo de lo que se puede hacer con la sintomatología depresiva en los servicios de atención primaria (Fleck et al, 2005; Goldberg y Huxley, 1980).

En España, la depresión tiene una amplia dispersión estadística que va de 5 a 20\% de prevalencia (Aragonés-Benaiges, 2005; Baca et al., 1999; Canals, Domenech, Carbajo y Blade, 1997; Gabarrón et al, 2002; López-lbor et al., 2000; Polaino-Lorente, Madiano et al., 1997; Vázquez-Barquero et al., 1987). En personas mayores de 64 años se ha hallado una prevalencia de 19,7\% (Urbina, Flores, Torres y Torrubias, 2007). Según la Encuesta Nacional de Salud 2006 de España (http://www.ine. es), un $14,7 \%$ de personas mayores de edad padece depresión, ansiedad u otros trastornos mentales. El porcentaje de adolescentes con factores de riesgo en barrios marginales es alrededor de un 30/50\% (Ezpeleta et al., 2007).

En Colombia, la prevalencia de la depresión también constituye un reto para la ciencia de la prevención. Algunos trabajos sobre población infantil presentan una prevalencia de la depresión de un 25,2\% (Vinaccia et al., 2006). Los estudios llevados a cabo con personas adultas (Gómez-Restrepo et al., 2004) son mucho más parecidos a los resultados de los trabajos de prevalencia de la depresión en la Unión Europea.

Los costos directos e indirectos de la depresión diagnosticada y tratada son muy significativos (Luppa, Heinridi, Angermeyer, Kömig y Riedel-Heller, 2007). A pesar de todo, no están satisfechas las necesidades de atención para el tratamiento y prevención de la salud mental en la Unión Eu- ropea (Alonso et al, 2007). Un 15\% de la personas que acuden a los centros de atención primaria de salud se podrían beneficiar de programas de prevención primaria de la depresión (Asociación Americana de Psiquiatría, 2004; Barry y Jenkis, 2006). La escuela también es un contexto adecuado para la promoción de la salud mental (Herman, Merrell y Reinke, 2004).

En la Estrategia en Salud Mental del Sistema Nacional de Salud 2006 se afirma: "No es fácil obtener información sobre las actividades de promoción y prevención en España. Las actuaciones de promoción de la salud mental y prevención de los trastornos mentales responden a iniciativas aisladas, desconectadas entre sí y de escasa difusión" (Ministerio de Sanidad y Consumo, 2007, p. 75). Es verdad: uno tiene la impresión de que la prevención puede esperar. De los estudios sociológicos realizados en los libros blancos de La depresión en España (Gabinete de Estudios Sociológicos Bernard Krief, 1982) y La calidad asistencial de la depresión en España (Gabinete de Estudios Sociológicos Bernard Krief, 1997) no se puede inferir un interés serio por las estrategias de prevención primaria de la depresión.

Además, en textos de psiquiatría (Barcia-Salorio, 2000; Vallejo-Ruiloba, 2006; Vallejo-Nájera, 1985) y psicología médica (López-lbor, Ortiz y López-lbor, 1999) ni siquiera se menciona el término “prevención” en el índice de materias. En otros, sólo aparece dos (Fuentenebro y Vázquez, 1990) o tres veces (Belloch, Sandín y Ramos, 1996). En los trabajos de Vallejo-Ruiloba y Gastó (1999) y López-lbor y Álamo (1998) también se olvida la prevención primaria en salud mental.

Los análisis bibliométricos disponibles también evidencian el poco interés que existe por la prevención de la depresión infantil (Diez-Zamorano, 2003). A fin de cuentas, todo lleva a admitir que en los libros de psiquiatría no parece haber lugar para la prevención (Cornes, Fernández-Ríos, Arauxo y Pedrejón, 2004; Martín-Águeda et al., 2006; Santo-Domingo, Baca, Carrasco-Perera y García-Ramos, 2002). Para los currículos de las facultades de psicología la ciencia de la prevención también es algo secundario e irrelevante. 


\section{Programas de prevención de la depresión}

Los factores de riesgo de la depresión unipolar son bien conocidos por todos los profesionales de la teoría y práctica de la ciencia de la prevención, ya que existen múltiples publicaciones que los describen. Dichos factores de riesgo son de carácter social y de procesamiento de información individual. En principio, no tendrían gran relevancia los factores psicobiológicos (Alloy y Riskind, 2004; Dobson y Dozois, en prensa); sin embargo, en muchas ocasiones, recurrir a mecanismos neurobiológicos es el recurso de los que no desean transformar la realidad sociomaterial.

La depresión tiene que ser una prioridad en salud pública, aunque Ayuso-Mateos (2004) se olvide de su prevención. Los fundadores de la psiquiatría (p.e., Kraepelin, 1988) también la marginaron. La bibliografía en lengua inglesa acerca de la prevención de la depresión es muy amplia, como se puede apreciar: Beardslee y Gladstone (2001); Dozois y Dobson (2004); Evans et al. (2005); Gillham, Shatté y Freres (2000); Hollon et al., (2002); Horowitz y Garber (2006); Merry, McDowell, Wild, Bir y Cunliffe (2004); Neil y Christensen (2007); Spence y Shortt (2007); Stice, Burton, Bearman y Rohde(2006); Sutton (2007).

En la presente revisión se observaron dos dimensiones. Por un lado, los fundamentos teóricos de los programas; éstos desarrollan, entre otras, las perspectivas de que las ideas irracionales causan y mantienen las perturbaciones emocionales (Ellis, 1980) y la relevancia de las creencias, en la tríada cognitiva (Beck, 1972). También son de resaltar las perspectivas de la teoría de la indefensión reformulada (Abramson, Seligman y Teasdale, 1978), de los estilos de respuesta (NolenHoeksema, 1991), de la psicología social cognitiva y autoeficacia (Bandura, 1997, 2002; Horowitz, Garber, Ciesla, Young y Mufson 2007; Pössel, Baldus, Horn, Groen y Hautzinger, 2005), del entrenamiento en habilidades socio-emocionales (Merrell, Gueldner y Tran, 2008; Sheffield et al., 2006; Vander Stoep, Weiss, McKnight, Beresford y Cohen, 2002; Young, Mufson y Davies, 2006) y de solución de problemas socio-existenciales (Nezu, Nezu y D'Zurilla, 2006).

En segundo lugar se observaron los contenidos: la relación entre cognición y afecto; estilos atribucionales; control de los pensamientos catastróficos acerca del futuro; habilidades de afrontamiento de los conflictos; asertividad y negociación constructiva; estrategias de solución de problemas; construcción de emociones positivas (como perdonar, sentido del humor, sentimiento de utilidad, etc.); sentido de trascendencia a través de las cosmovisiones religiosas/espirituales; estructura personal y social de valores; sistema de apoyo social, etc.

En todos los casos se deben buscar estrategias de afrontamiento centradas en la solución de problemas y solicitar ayuda cuando sea necesario. Los programas de prevención tienen y deben realzar el self (Sedikides \& Strube, 1997) y las emociones positivas (Tracy, Robins y Tangney, 2007). El self agéntico viene a ser similar al de autoeficacia (Little, Snyder y Wehmeyer, 2006; Shoda, Cervone y Downey, 2007). En general, se acepta que la relación entre características positivas de personalidad y salud son positivas (Newton-Howes, Tyrer y Johnson, 2006; Vollrath, 2006). Todo proceso de prevención va de lo interpersonal a lo intraindividual. Los recursos para el crecimiento postraumático comienzan por ser interindividuales; sólo posteriormente devienen intraindividuales. Es el origen social de los recursos psicológicos para la prevención.

En cuanto a programas, cabe destacar, por un lado, intervenciones preventivas universales como el Problem solving for live (Spence, Sheffield y Donovan, 2005); el Resource adolescent program (Dadds y Roth, en prensa; Harnett y Dadds, 2004; Shochet y Ham, 2004); los programas Strong kids (Merrell, Parisi y Whitcomb, 2007) y, por otro, los programas de prevención específicos como los dirigidos a hijos de padres depresivos (Beardslee, Wright y Salt, 1997; Clarke et al., 1995; Clarke et al., 2001). Así mismo, son de resaltar las diferentes versiones del Penn prevention program (Cardemil, Reivich, Beevers, Seligman y James, 2007; Gillham et al., 2007; Gillham, Hamilton, Freres, 
Patton, Gallop, 2006; Gillham y Reivich, 1999; Seligman, Reivich, Jaycox y Gillham, 1995) y el San Francisco depression prevention research project (Muñoz, 1987; Muñoz y Ying, 1993).

Entre las investigaciones longitudinales merece especial mención el Simmons longitudinal study (Paradis, Reinherz, Giaconia y Fitzmaurice, 2006) y el Temple-Wisconsin cogntive vulnerability to depression (Alloy y Abramson, 1999). También se juzgó relevante tener en cuenta, entre otros muchos, el Gatehouse project (Bond et al., 2004; Patton, Bond Butler y Glover, 2003; Patton, Glover, Bond, Butler y Bowes, 2000), el Beyondblue: the national depression initiative (Hickle, 2001; Spence et al., 2005) y el FRIENDS (Stallard, Simpson, Anderson, Hibbert y Osborn, 2007). Pues, previniendo la ansiedad, se contribuye a la reducción de la incidencia de la depresión (Barrett, Byford y Knapp, 2005; Farrell y Barrett, 2007; Feldner et al., 2004; Flannery-Schroeder, 2006; Turner y Dadds, 2001; Wright, K. D., Stewart, S. H., Finley, G. A. y Buffett-Jerrott, S. E., 2007). Es la cuestión de la coprevención (Krueger y Markon, 2006).

La prevención de la depresión es posible; sin embargo, los efectos de los programas no son siempre positivos. Cuando consiguen resultados esperanzadores, éstos no son permanentes. Se debe hacer lo que realmente sea posible; que, por el momento, es poco. Pero cuando se contribuye a reducir el sufrimiento humano, ese poco puede ser mucho. Además, los programas de prevención de la depresión tienen mejores resultados que las intervenciones placebo (Merry et al., 2004).

\section{Problemas para la prevención primaria basada en la evidencia en España}

Los programas de prevención deben ser socialmente sensibles y relevantes en la cultura en la que se aplican. Un buen ejemplo de ello es el Family Coping Skills Program (Cardemil, Kim, Pinedo y Miller, 2005). La prevención forma parte del espíritu de los tiempos, pero tiene y debe estar fundamentada en realidades (Jané-Llopis, et al., 2005; Jané-Llopis, Hosman, Jenkins y Anderson, 2003, 2005). La práctica de la prevención primaria debe llevarse a cabo con cautela (Harrington y Clark, 1998; Merry y Spence, 2007; Paykel, Brugha y Fryers, 2005, p. 419; Wells et al., 2003). En Europa son de destacar la European Alliance Against Depression (http://www.eaad.net) y la European Network for Mental Health Promotion and Mental Disorder Prevention (http://www. imhpa.net) que pueden y deben hacer mucho.

Los problemas de la prevención de la depresión en España se pueden dividir en dos grandes apartados. Por un lado, tenemos una serie de cuestiones generales. Son las siguientes:

1. Poca evaluación basada en la evidencia de los sujetos en riesgo de depresión (Hunsley y Mash, 2007).

2. Conflictos entre paradigmas y entre disciplinas académicas para describir, comprender y planificar programas de prevención.

3. Inadecuada utilización de la estadística y un excesivo énfasis en lo cuantitativo, olvidando lo cualitativo.

4. Aceptación acrítica del construccionismo social en la prevención de la depresión.

5. Utilización abusiva del concepto de comunidad. La psicología comunitaria deviene académicamente sugerente (Reich, Riemer, Prillelttensky y Montero, 2007), pero prácticamente irrelevante.

6. Confusión teórica: en ella se utilizan, por un lado, los conceptos de prevención primaria y secundaria y, por otra, los de prevención universal y selectiva. Pues lo que se denomina prevención terciaria y específica no constituye, en la práctica, una estrategia de prevención. También es necesario tener en cuenta los trabajos de Caplan $(1985,1993)$ y Cowen (1983). Véase, por ejemplo, The Journal of Primary Prevention (28 (1), 2007). 
En segundo lugar, se debe tener en cuenta una serie de cuestiones específicas en España. Son las siguientes:

1. Falta de profesionales de la práctica de la prevención primaria.

2. Deficiencias en el currículo universitario.

3. Recelo entre psiquiatras y psicólogos clínicos.

4. Presupuesto económico deficiente.

5. Desinterés político-sanitario y apatía de la población.

6. ¿Dónde aplicar los programas de prevención?

\section{7. ¿Quién debe aplicar los programas?}

Según Jané-Llopis (2002, 2004), los programas de prevención de la enfermedad mental y promoción de la salud mental son mucho más eficaces cuando los aplican profesionales competentes de la salud. Esto sugiere-según Jané-Llopis (2004)que "la prevención debería incluirse en los sistemas de atención primaria y secundaria” ( $p$. 72). La evidencia empírica disponible lleva a la conclusión de que la prevención de los desórdenes mentales necesita ser una parte integral de las políticas de promoción de las salud pública (Saxena, Jané-Llopis y Hosman, 2006). El proceso de trasladar la teoría a la práctica es uno de los problemas teórico-políticos de la prevención de la depresión (Glasgow y Emmons, 2007; Izard, 2002).

\section{Conclusión e implicaciones futuras}

En España, la psiquiatría y la psicología olvidan la prevención primaria de la depresión unipolar. Albee (2005) llama a una revolución en la prevención de los trastornos mentales. No se busca tanto; simplemente, nos conformaríamos con la utilización del sentido común, lo cual ni siquiera se hace. La tradición de la psiquiatría en este país es diagnosticar, tratar y rehabilitar. La de la psicología es más confusa, pues, al mismo tiempo que busca y todavía anhela su identidad social, ha marginado la prevención.

En función de todo lo anterior, no se puede ser demasiado optimista acerca del futuro de la prevención de la depresión unipolar en España. La cuestión no es tanto que no se conozca qué hacer, sino que realmente no se pone en práctica el conocimiento del que se dispone. En el documento Estrategia en salud mental del Sistema Nacional de Salud 2006 (Ministerio de Sanidad y Consumo, 2007) se establecen una serie de recomendaciones para la mejora de la prevención de la enfermedad mental. Esperemos que se lleven a la práctica con las personas de todas las clases sociales (Patel et al., 2007).

También se pueden tener en cuenta las posibilidades que ofrece internet. Un buen ejemplo de ello es el programa de prevención de la depresión y la ansiedad de Seligman, Schulman y Tryon (2007) y de Christensen y Griffiths (2002). También Muñoz y Mendelson (2005).

\section{Referencias}

Abramson, L.Y., Seligman, M.E. \& Teasdale, J.D. (1978). Learned helplessness in humans: Critique and reformulation. Journal of Abnormal Psychology, 87, 49-74.

Albee, G.W. (2005). Call to prevention in the prevention of emotional disorders. Ethical Human Psychology and Psychiatry, 7, 37-44.

Alloy, L.B. \& Abramson, L. Y. (1999). The templewisconsin cognitive vulnerability to depression project: Conceptualbackground, design and methods. Journal of Cognitive Psychotherapy, 13, 227-262. 
Alloy, L.B. \& Riskind, J.H. (eds.). (2004). Cognitive vulnerability to emotional disorders. Hillsdale: Erlbaum.

Alonso, J., Codony, M., Kovess, V., Angerrmeyer, M.C., Katz, S.J., Haro, J.M., Girolamo, G., Graaf, R., Demyttenaere, K., Vilagut, G., Almansa, J., Lépine, J.P., Brugha, T.S. (2007). Population level of unmet need for mental healthcare in Europe. British Journal of Psychiatry, 190, 299-306.

Aragonés-Benaiges, E. (2005). Estudio de los trastornos depresivos en la atención primaria de salud. Revista de Psiquiatría de la Facultad de Medicina de Barcelona, 32, 30-37.

Asociación Americana de Psiquiatría (2004). DSMIV-TR: AP: Atención Primaria. Barcelona: Masson.

Ayuso-Mateos, J.L., Vázquez-Barquero, L., Dowrick, C., Lehtinen, V., Dalgard, O.S., Casey, P., Wilkinson, C., Lasa, L., Page, H., Dunn, G., Wilkinson, G. \& The ODIN Group (2001). Depressive disorders in Europe: Prevalence Figures from the ODIN study. British Journal of Psychiatry, 179, 308-316.

Ayuso-Mateos, J. L. (2004). Depresión: una prioridad en salud pública. Medicina Clínica, 123, 191-196.

Baca, E. \& Lázaro, J. (eds.). (2003). Hechos y valores en psiquiatría. Madrid: Triacastela.

Baca, E., Sáiz, J., Agüera, L.F., Caballero, L., Fernández-Liria, A., Ramos, J.A. et al. (1999). Prevalencia de los trastornos psiquiátricos en atención primaria usando el cuestionario Prime MD. Atención Primaria, 23, 275-279.

Bandura, A. (1997). Self-eficacy. Nueva York: W.H. Freeman.

Bandura, A. (2002). Growing primacy of human agency in adaptation and change in the electronic era. European Psychologist, 7, 2-16.
Barcia-Salorio, D. (1987). La psicoterapia como fundamento antropológico. En D. Barcia (ed.). Psiquiatría antropológica (pp. 189-212). Murcia: Servicio de Publicaciones de la Universidad de Murcia.

Barcia-Salorio, D. (ed.). (2000). Tratado de psiquiatría. Madrid: Arán.

Barrett, B., Byford, S. \& Knapp, M. (2005). Evidence of cost-effective treatment for depression: A systematic review. Journal of Affective Disorders, 84, 1-13.

Barry, M. \& Jenkis, R. (2006). Implementing mental health promotion. Oxford: Elsevier.

Beardslee, W. R. \& Gladstone, T.R.G. (2001). Prevention of childhood depression: Recent findings and future prospects. Biological Psychiatry, 49, 1101-1110.

Beardslee, W., Wright, E. M. \& Salt, P. (1997). Examination of children's responses to two preventive intervention strategies over time. Journal of the American Academy of Child and Adolescent Psychiatry, 36, 196-204.

Beck, A. (1972). Depression. Philadelphia: University of Pennsylvania Press.

Belloch, A., Sandín, B., \& Ramos, F. (eds.). (1996). Manual de psicopatología (vols. I-II). Madrid: McGraw-Hill.

Bernal, M., Haro, J.M., Bernert, S., Brugha, T., de Graaf, R., Brufffaerts, R., Lëpine, J.P., de Girolamo, G., Vilagut, G., Gasquet, I., Torres, J.V., Kovess, V., Heider, D., Neeleman, J., Kessler, R., Alonso, J. (2007). Risk factors for suicidality in Europe: Results from the ESMED study. Journal of Affective Disorders, 101, 2734.

Berrios, G. \& Olivares, J. (1998). ¿Por qué la historia debería ser una ciencia básica para la psiquiatría?. Psiquiatría Biológica, 2, 70-83. 
Bond, L., Patton, G., Glover, S., Carlin, J.B., Butler, H., Thomas, L. \& Bowes, G. (2004). The Gatehouse project: A multilevel school intervention affect emotional wellbeing and health risk behaviours?. Journal of Epidemiology and Community Health, 58, 997-1003.

Canals, J., Domenech, E., Carbajo, J. \& Blade, J. (1997). Prevalence of DSM-III-R and ICD-10 psychiatric disorders in a Spanish population of 18-Year-Old. Acta Psychiatrica Scandinavica, 96, 287-294.

Caplan, G. (1985). Principios de psiquiatría preventiva. Barcelona: Paidós.

Caplan, G. (1993). Aspectos preventivos en salud mental. Barcelona: Paidós.

Cardemil, E. V., Reivich, K.J., Beevers, C.G., Seligman, M. E.P. \& James, J. (2007). The prevention of depressive symptoms in low-income, minority children: Two-year follow-up. Behaviour Research and Therapy, 45, 313-327.

Cardemil, E., Kim, S., Pinedo, T.M. \& Miller, I.W. (2005). Developing a culturally appropriate depression prevention program: The family coping skills program. Cultural Diversity and Ethnic Minority Psychology, 11, 99-112.

Castilla del Pino, C. (1966). Un estudio sobre la depresión. Barcelona: Península.

Christensen, H. \& Griffiths, K.M. (2002). The prevention of depression using the internet. The Medical Journal of Australia, 177, S122-S125.

Clarke, G.N., Hawkins, W., Murphy, M., Sheeber, L.B., Levinsohn, P.M. \& Seeley, J.S. (1995). Targeted prevention of unipolar depressive disorder in an at-risk sample of high school adolescents: A randomized trial of a group cognitive Intervention. Journal of the American Academy of Child and Adolescent Psychiatry, 34, 312-321.
Clarke, G.N., Hornbrook, M., Lynch, F., Polen, M., Gale, J., Beardslee, W., O'Connor, E. \& Seeley, J. (2001). A randomized trial of a group cognitive intervention for preventing depression in adolescent offspring of depressed parents. Archives of General Psychiatry, 58, 1127-1134.

Cornes, J.M., Fernández-Ríos, L., Arauxo, A., Pedrejón, C. (2004). Ciencia de la prevención: fundamentos y perspectivas. Implicaciones en psicología. Revista de Psiquiatría de la Facultad de Medicina de Barcelona, 3, 86-95.

Cowen, E.L. (1983). Primary prevention in mental health: Past, present and future. En: R.D. Felner, L.A. Jason, J.N. Moritsugu \& S. Farber (eds.). Preventive psychology (pp. 11-30). Nueva York: Oxford University.

Dadds, M.R. \& Roth, J.H. (en prensa). Prevention of anxiety disorders: Results of a universal trial with young children. Journal of Child and Family Studies.

Diez-Zamorano, M.A. (2003). Análisis bibliométrico sobre depresión infantil en España. Revista Internacional de Psicología Clínica y de la Salud/International Journal of Clinical and Health Psychology, 3, 645-653.

Dobson, K.S. \& Dozois, D. (eds.) (en prensa). Risk factors for depression. Nueva York: Elsevier.

Dozois, D. \& Dobson, K.S. (eds.). (2004). The prevention of anxiety and depression. Washington: APA Press.

Ellis, A. (1980). Razón y emoción en psicoterapia. Bilbao: Desclée de Bouwer.

Evans, D.L., Foa, E., Gur, R., Hendrin, H., O’Brien, C., Seligman, M.E.P. \& Walsh, B.T. (eds.). (2005). Treating and preventing adolescent mental health disorders. Nueva York: Oxford University. 
Ey, H. (1967). La conciencia. Madrid: Gredos.

Ezpeleta, L., Guillamón, N., Granero, R., De la Osa, N., Doménech, J.M. \& Moya, I. (2007). Prevalence of mental disorders in children and adolescents from Spanish slum. Social Science and Medicine, 64, 842-849.

Farrell, L.J. \& Barrett, P.M. (2007). Prevention of childhood emotional disorders: Reducing the Burden of Suffering Associated with Anxiety and Depression. Child and Adolescent Mental Health, 12, 58-65.

Feldner, M.T., Zvolensky, M.J. \& Schmidt, N.B. (2004). Prevention of anxiety psychopathology: A critical review of the empirical literature. Clinical Psychology: Science and Practice, 11, 405-424.

Flannery-Schroeder, E.C. (2006). Reducing anxiety to prevent depression. American Journal of Preventive Medicine, 31 (6S1), S136-S142.

Fleck, M.P.A., Simon, G., Herrman, H., BushneII, D., Martin, M. \& Patrick, D. (2005). Major depression and its correlates in primary care settings in six countries. 9-Moth follow-up study. British Journal of Psychiatry, 186, 4147.

Fuentenebro, F. \& Vazquez, C. (eds.). (1990). Psicología médica y psiquiatría (vols. I-II). Madrid: McGraw-Hill.

Gabarrón, E., Vidal, J.M., Haro, J.M., Boix, I., Jover, A. \& Arenas, M. (2002). Prevalencia y detección de los trastornos depresivos en atención primaria. Atención Primaria, 29, 329-337.

Gabinete de estudios sociológicos Bernard Krief. (1982) Estudio sociológico Libro Blanco. La depresión en España. Madrid: Publisher.

Gabinete de estudios sociológicos. (1997) Estudio sociosanitario Libro Blanco "La calidad asistencial de la depresión en España”. Madrid: Publisher.
Gillham, J.E. y Reivich, K.J. (1999. Prevention of depressive symptoms in school children: $A$ research update. Psychological Science, 10, 461-462.

Gillham, J.E., Freres, D.R., Chaplin, T.M., Shatté, A.J., Samuels, B., Elkon, A.G.L., Litzinger, S., Lascher, M., Gallop, R. \& Seligman, M.E.P. (2007). School-based prevention of depressive symptoms: A randomized controlled study of the effectiveness and specificity of the penn resiliency program. Journal of Consulting and Clinical Psychology, 75, 9-19.

Gillham, J. E., Shatté, A.J. y Freres, D.R. (2000). Depression prevention: A review of cognitivebehavioral and family interventions. Applied and Preventive Psychology, 9, 63-88.

Gillham, J. E., Hamilton, J., Freres, D. R., Patton, K., Gallop, R. (2006). Preventing depression among early adolescents in the primary care settings: A randomized controlled study of the penn resiliency program. Journal of $A b$ normal Child Psychology, 34, 203-219.

Glasgow, R.E. y Emmons, K.M. (2007). How can we increase translation of research into practice? Types of evidence needed. Annual Review of Public Health, 28, 413-433.

Goldberg, D. y Husley, P. (1980). Mental illness in the community. London: Tavistock.

Gómez-Restrepo, C., Bohórquez, A., Pinto, D., Gil, J., Rondón, M. \& Díaz-Granados, N. (2004). Prevalencia de depresión y factores asociados con ella en la población colombiana. Revista Panamericana de Salud Pública, 16, 378-386.

Harnett, P.H. y Dadds, M.R. (2004). Training School personnel to implement a universal school-based prevention of depression program under real-world conditions. Journal of School Psychology, 42, 343-357. 
Harrington, R. \& Clark, A. (1998). Prevention and early intervention for depression in adolescence and early adult life. European Archives of Psychiatry and Clinical Neuroscience, 248, 32-45.

Herman, K.C., Merrell, K.W. y Reinke, W.M. (2004). The role of school psychology in prevention depression. Psychology in the School, 4, 763775.

Hickle, I. (2001). Beyondblue: The national depression initiative. Australian Psychiatry, 9, 147-150.

Hollon, S.D., Muñoz, R.F., Barlow, D.H., Beardslee, W.R., Bell, C.C., Bernal, G., Clarke, G.N., Franciosi, L.P., Kazdin, A.E., Kohn, L., Linehan, M.M., Markowitz, J.C., Miklowitz, D.J., Persons, J.B., Niederehe, G. \& Sommers, D. (2002). Psychosocial intervention development for the prevention and treatment of depression: Promoting innovation and increasing access. Biological Psychiatry, 52, 610-630.

Horowitz, J.L. \& Garber, J. (2006). The prevention of depressive symptoms in children and adolescents: a meta-analytic review. Journal of Consulting and Clinical Psychology, 74, 401-415.

Horowitz, J.L., Ciesla, J.A., Young, J.F. \& Mufson, L. (2007). Prevention of depression symptoms in adolescents: A randomized trial of cognitive-behavioral and interpersonal prevention programs. Journal of Consulting and Clinical Psychology, 75, 693-706.

Hunsley, J. \& Mash, E.J, (2007). Evidence-based assessment. Annual Review of Clinical Psychology, 3, 57-79.

Izard, C. (2002). Translating emotion theory and research into preventive interventions. Psychological Bulletin, 128, 796-824.

Jané-Llopis, E. (2002). What makes the ounce of prevention effective. Nijmegen: Nijmegen University.
Jané-Llopis, E. (2004). La eficacia de la promoción de la salud mental y la prevención de los trastornos mentales. Revista de la Asociación Española de Neuropsiquiatría, 24, 67-77.

Jané-Llopis, E. (2006). From evidence to practice: mental health promotion effectiveness. Australian e-Journal for the Advancement of Mental Health, 5, 1-11.

Jané-Llopis, E. \& Anderson P. (2005). Mental health promotion and mental disorder prevention: $A$ policy for Europe. Nijmegen: Radboud University Nijmegen

Jané-Llopis, E., Barry, M., Hosman, C. \& Patel, V. (2005). Mental health promotion works: A review. Promotion and Education (suppl), 2, 9-25.

Jané-Llopis, E., Hosman, C., Jenkins, R. \& Anderson, P. (2003). Predictors of efficacy in depression prevention programmes. Metaanalysis. British Journal of Psychiatry, 183, 384-397.

Jaspers, K. (1977) Psicopatología general. Buenos Aires: Beta.

Kessler, R.C. (2007). The global burden of anxiety and mood disorders. Putting ESEMeD findings into perspective. Journal of Clinica Psychiatry, 68 (suppl 2), 10-19.

King, M., Weich, S., Torres-González, F., Svab, I., Maaroos, H.I., Neeleman, J., Xavier, M., Morris, R., Walker, C., Bellón-Saameño, J.A., Moreno-Küstner, B., Rotar, D., Rifel, J., Aluoja, A., Kalda, R., Geerlings, M.I., Carraça, I., Caldas de Almeida, M., Vicente, B., Saldivia, S., Rioseco, P. \& Nazareth, I. (2006). Prediction of depression in european general practice attendees: The Predict study. BCM Public Health, 6, 1-8.

Kraepelin, E. (1988) Introducción a la clínica psiquiátrica. Madrid: Nieva. 
Krueger, R.F. \& Markon, K.E. (2006). Reinterpreting comorbidity: A model-based approach to understanding and classifying psychopathology. Annual review of clinical psychology, 2,111-133.

Little, T.D., Snyder, C.R. \& Wehmeyer, M. (2006). The agentic self: On the nature and origins of personal agency across the life Spain. En: D.M. Mroczek, T.D. Little (eds.). Handbook of personality development. Mahwah: Erlbaum.

López-lbor, J.J. y Álamo, C. (1998). Quince años de asistencia a la depresión en España. Estudio sociosanitario "Libro Blanco" 1982 vs. “Libro Blanco" 1997. Alcalá de Henares: Servicio de Publicaciones de la Universidad de Alcalá de Henares.

López-lbor, J.J., Álamo, C., López-Muñoz, F., Cuenca, E., Rubio, G. \& Otero, F.J. (2000). Evolution of the management of depression in Spain from the psychiatrist's perspective. A comparative analysis: 1997 vs 1982. European Psychiatry, 15, 362-369.

López-Ibor, J.J., Ortiz, T. y López-lbor, M.I. (1999). Lecciones de psicología médica. Barcelona: Masson.

Luppa, M., Heinrich, S., Angermeyer, M.C., König, H.H and Riedel-Heller, S. G. (2007). Cost-ofillness studies of depression: A systematic review. Journal of Affective Disorders, 98, 29-43.

Martín-Águeda, B., López-Muñoz, F., Rubio, G., García-García, P., Silva, A. \& Álamo, C. (2006). Current situation of depression healthcare in Spain: Results of a psychiatrist'survey. European Journal of Psychiatry, 20, 211-223.

McCracken, C., Dalgard, O.S., Ayuso-Mateos, J.L., Casey, P., Wilkinson, G., Lehtinen, V. \& Dowrick, C. (2006). Health service use by adults with depression: Community survey in five european countries. Evidence from the Odin study. British Journal of Psychiatry, 189, 161167.
Merrell, K.W., Parisi, D. \& Whitcomb, S. (2007). Strong start. Baltimore: Paul H. Brookes.

Merrell, K.W., Gueldner, B.A. \& Tran, O.K. (2008). Social and emotional learning: A school-wide approach to intervention for socialization, friendship problems, and more. En: B. Doll \& J.A. Cummings (eds.). Transforming school mental health services. Thousand Oaks: Sage.

Merry, S.N., Hetrick, S., McDowell, H. \& Bir, J. (2004). The effectiveness of psychological and/or educational interventions for the prevention of depression in children and adolescents. The Cochrane Database of Systematic Reviews (1).

Merry, S.N., McDowell, H., Wild, C., Bir, J. \& Cunliffe, R. (2004). A randomized placebocontrolled trial of a school-based depression prevention program. Journal of the American Academy of Child and Adolescent Psychiatry, 43, 538-547.

Merry, S.N., \& Spence, S.H. (2007). Attempting to prevent depression in youth: A systematic review of the evidence. Early Intervention in Psychiatry, 1, 128-137.

Ministerio de Sanidad y Consumo (2007). Estrategia en salud mental del Sistema Nacional de Salud, 2006. Madrid: Ministerio de Sanidad y Consumo.

Muñoz, R. (ed.). (1987). Depression prevention. Londres: Hemisphere.

Muñoz, R.F. \& Mendelson, T. (2005). Toward evidence-based interventions for diverse populations: The San Francisco General Hospital Prevention and Treatment Manuals. Journal of Consulting and Clinical Psychology, 73, 790-799.

Muñoz, R.F. \& Ying, Y.W. (1993). The prevention of depression. Baltimore: Johns Hopkins University Press. 
Murray, C.J.L. \& López, A.L. (1997). Alternative projections of mortality \& disability by cause 1990-2020: Global burden of disease study. Lancet, 349, 1498-1504.

Murphy, J.M., Sobol, A.M., Neff, R.K., Oliver, D.C. \& Leighton, A.H. (1984). Stability of prevalence: depression and anxiety disorders. Archives of General Psychiatry, 41, 990-997.

Neil, A.L. \& Christensen, H. (2007). Australian school-based prevention and early intervention programs for anxiety and depression: A systematic review. Medical Journal of Australia, 186, 305-308.

Newton-Howes, G., Tyrer, P. \& Johnson, T. (2006). Personality disorders and the outcome of depression: Meta-analysis of published studies. British Journal of Psychiatry, 188, 13-20.

Nezu, A.M., Nezu, C.M. \& D’Zurilla, T. (2006). Solving life's problems. Berlín: Springer.

Nolen-Hoeksema, S. (1991). Responses to depression and their affects on the duration of depressive episodes. Journal of Abnormal Psychology, 100, 569-582.

Owen, G. \& Harland R (eds.) (2007). Editor's Introduction: Theme issue on phenomenology and psychiatry for the 21st Century. Taking phenomenology seriously. Schizophrenia Bulletin, 33, 105-173.

Paradis, A.D., Reinherz, H.Z., Giaconia, R.M. \& Fitzmaurice, G. (2006). Major depression in the transition to adulthood: The impact of active and past depression on young adult functioning. Journal of Nervous and Mental Disease, 194, 318-323.

Patel, V., Araya, R., Chatterjee, S., Chisholm, D., Cohen, A., De Silva, M., Hosman, C., McGuire, H., Rojas, G. \& Van Ommeren, M. (2007). Treatment and prevention of mental disorders in low-income and middle-income countries. Lancet, 370, 991-1005.
Patton, G., Bond, L., Butler, H. \& Glover, S. (2003). Changing schools, changing health? Design and implementation of the gatehouse project. Journal of Adolescence Health, 33, 231-239.

Patton, G., Glover, S., Bond, L., Butler, H. \& Bowes, G. (2000). The gatehouse project: A systematic approach to mental health promotion in schools. Australian and New Zealand Journal of Psychiatry, 34, 586-593.

Paykel, E.S., Brugha, T. \& Fryers, T. (2005). Size and burden of depressive disorders in Europe. European Neuropsychopharmacology, 15, 411-423.

Polaino-Lorente, A., Mediano, M.L., Martínez, J. \& Arias, R. (1997). Epidemiological study of the symptomatology of children depression in madrid school-age population. Anales Españoles de Pediatría, 46, 344-350.

Pössel, P., Baldus, C., Horn, A.B., Groen, G. \& Hautzinger, M. (2005). Influence of general self-efficacy on the effects of a school-based universal primary prevention program of depressive symptoms in adolescents: A randomized and controlled follow-up study. Journal of Child Psychology and Psychiatry, 46, 982-994.

Reich, S.M., Riemer, M., Prilleltensky, I. \& Montero, M. (2007). International Community Psychology. Nueva York: Springer.

Santo-Domingo, J., Baca, E., Carrasco-Perera, J.L. \& García-Camba, E. (2002). Manual de psiquiatría. Barcelona: Ars Medica.

Saxena, S., Jané-Llopis, E. \& Hosman, C. (2006). Prevention of mental and behavioral disorders: Implications for policy and practice. World Psychiatry, 5, 5-14.

Sedikides, C. \& Strube, M.J. (1997). Self-evaluation: to thine own self be good, to thine own self be sure, to thine own self be true and to 
thine own self be better. Advances in Experimental Social Psychology, 29, 206-269.

Seligman, M.E.P., Reivich, K., Jaycox, L. \& Gillham, J. (1995). The optimistic child. Boston: Houghton Mifflin.

Seligman, M.E.P., Schulman, P. \& Tryon, A.M. (2007). Group prevention of depression and anxiety symptoms. Behaviour Research and Therapy, 45, 1111-1126.

Sheffield, J.K., Spence, S.H., Rapee, R.M., Kowalenko, N., Wignall, A., Davis, A. \& McLoone, J. (2006). Evaluation of universal indicated and combined cognitive-behavioral approaches to the prevention of depression among adolescents. Journal of Consulting and Clinical Psychology, 74, 66-79.

Shochet, I.M. \& Ham, D. (2004). Universal schoolbased approaches to preventing adolescent depression: Past findings and future directions of the resourceful adolescent program. International Journal of Mental Health Promotion, 6, 17-25.

Shoda, Y., Cervone, D. \& Downey, G. (eds.). (2007). Persons in context. Nueva York: Guilford Press.

Spence, S., Burns, J., Boucher, S., Graetz, B, Kay, D., Patton, G. \& Sawyer, M. (2005). The beyondblue schools research initiative: Conceptual framework and intervention. Australian Psychiatry, 13, 159-164.

Spence, S. \& Shortt, A.L. (2007). Research review: Can we justify the widespread dissemination of universal, school-based interventions for the prevention of depression among children and adolescents?". Journal of Child Psychology and Psychiatry, 48, 526-542.

Spence, S., Sheffield, J.K. \& Donovan, C.L. (2005). Long-term outcome of a school-based, universal approach to prevention of depression in adolescents. Journal of Consulting and Clinical Psychology, 73, 160-167.
Stallard, P, Simpson, N., Anderson, S., Hibbert, S. \& Osborn, C. (2007). The Friends emotional health programme: Initial findings from a school-based project. Child and Adolescent Mental Health, 12, 32-37.

Stice, E., Burton, E, Bearman, S.K. \& Rohde, P. (2006). Randomized trial of a brief depression prevention program: An Elusive search for a psychosocial placebo control condition. Behavioral Research and Therapy, 45, 863876.

Sutton, J.M. (2007). Prevention of depression in youth: A qualitative review and future suggestions. Clinical Psychology Review, 27, 552571.

Tracy, J.L., Robins, R.W. \& Tangney, J.P. (eds.). (2007). The Self-Conscious Emotions. Nueva York: Guilford.

Turner, C. \& Dadds, M.R. (2001). Prevention and treatment strategies. En: F. Fincham, \& $\mathrm{H}$. Grych (eds.). Child development and interparental conflict (pp. 387-416). Cambridge: Cambridge University Press.

Tylee, A. (2000). Depression in Europe: Experience from the Depres II Survey. European Neuropsychopharmacology, 10 (suppl. 4), S445S448.

Urbina, J.R., Flores, J.M., Torres, S.L. \& Torrubias, R.M. (2007). Síntomas depresivos en personas mayores. Prevalencia y factores de riesgo. Gaceta Sanitaria, 21, 37-42.

Vallejo-Nájera, J.A. (1985). Introducción a la psiquiatría. Barcelona: Científico-Médica.

Vallejo-Ruiloba, J. \& Gastó, C. (eds.). (1999) Trastornos afectivos. Barcelona. Masson.

Vallejo-Ruiloba, J. V. (2006). Introducción a la psicopatología y la psiquiatría. Barcelona: Masson. 
Vander Stoep, A., Weiss, N.S., McKnight, B., Beresford, S.A.A. \& Cohen, P. (2002). Which measure of adolescent psychiatry disorderdiagnosis, number of symptoms or adaptive functioning-Best predicts adverse young adult outcomes. Journal of Epidemiology and Community Health, 56, 56-65.

Vázquez-Barquero, J.L., Díez-Manrique, J.F., Aldama, J., Samaniego, C., Menéndez, J. \& Mirapeix, C. (1987). Community mental health survey in Cantabria: A general description of morbidity. Psychological Medicine, 17, 227241.

Vinaccia, S., Gaviria, A.M., Atehortúa, L.F., Martínez, P.H., Trujillo, C. \& Quiceno, J.M. (2006). Prevalencia de la depresión en niños escolarizados entre 8 y 12 años del oriente antioqueño a partir del "Child depression inventory" (CDI). Diversitas: Perspectivas en Psicología, 2, (2), 217-227.

Vollrath, M.E. (ed.). (2006). Handbook of personality and health. Chichester: Wiley.
Wells, J., Barlow, J. \& Stewart-Brown, S. (2003). A systematic review of universal approaches to mental health promotion in schools. Health Education Journal, 103, 197-220.

Wittchen, H.U. \& Jacobi, F. (2005). Size and burden of mental disorders in Europe - A critical review and appraisal of 27 studies. European Neuropsychopharmacology, 15, 357-376.

World Health Organization (2001). The World health report, 2001. Ginebra: WHO.

World Health Organization (2004). Prevention of Mental Disorders. Ginebra: WHO.

Wright, K.D., Stewart, S.H., Finley, G.A. \& Buffett-Jerrott, S.E. (2007). Prevention and intervention strategies to alleviate preoperative anxiety in children. A critical review. Behavior Modification, 31, 52-79.

Young, J.F., Mufson, L. \& Davies, M. (2006). Efficacy of interpersonal psychotherapy-adolescent skills training: An indicated preventive intervention for depression. Journal of Child Psychology and Psychiatry, 47, 1254-1262. 